\title{
Análisis del proceso de evaluación del Trabajo Fin de Grado en las nuevas titulaciones
}

\author{
An analysis of the Final Dissertation Assessment Process in new \\ degrees
}

Ma Jesús Bonilla Priego (mariajesus.bonilla@urjc.es)

Laura Fuentes Moraleda (laura.fuentes@urjc.es)

Catalina Vacas Guerrero(catalina.vacas@urjc.es)

Trinidad Vacas Guerrero (trinidadvacas@urjc.es)

Universidad Rey Juan Carlos de Madrid (España)

http://dx.doi.org/10.12795/EDUCADE.2012.i03.02

\begin{abstract}
RESUMEN: El R.D 1393/2007 de 29 de octubre establece que las enseñanzas de Grado culminarán con la elaboración y defensa de un Trabajo Fin de Grado (TFG), el cual estará orientado a la evaluación de competencias asociadas al título. Los títulos de grado que comenzaron su andadura en el curso 2008/09, culminarán en el próximo curso, con la presentación de los alumnos de un trabajo fin de grado. Aunque existen titulaciones con gran tradición en la elaboración de un trabajo o proyecto fin de carrera; no es este el caso en otras titulaciones vinculadas a las ciencias sociales.

Por ello, el objeto de este trabajo consiste en seleccionar las competencias transversales objeto de evaluación, los agentes evaluadores; junto con el conjunto de indicadores empleados para medir el grado de cumplimiento con el que el alumno alcanza las competencias objeto de evaluación por el trabajo fin de grado.
\end{abstract}

PALABRAS CLAVE: Trabajo fin de grado, competencias transversales, agentes evaluadores, indicadores.

ABSTRACT: According to October 29th 1393/2007 R.D. all Spanish new degrees will end up with a final year project which is aimed at evaluating the degree competencies. Those degrees started in 2008/09 school year will be finished in 2011/12 and therefore in 2012 students will be able to present their final projects. Although, some degrees have traditionally included a final Project as part of their curriculum, this has not been the case for Spanish social sciences degrees.

Therefore, this paper shows the process undertaken to select those cross-curricular competences which will be assessed with the degree in Tourism final project, which will be in charge of evaluating the projects, as well as a set of indicators or assessment criteria to appraise the level of achievement of cross-curricular competencies.

KEYWORDS: Final year project, cross-curricular competencies, indicators, evaluators.

\section{INTRODUCCIÓN}

El Real Decreto 1393/2007 de 29 de octubre establece que las enseñanzas de Grado culminarán con la elaboración y defensa de un Trabajo Fin de Grado (TFG), el cual deberá realizarse en la fase final del plan de estudios y estará orientado a la evaluación de competencias asociadas al título.

Experiencia. Recibido: 04-09-12 - Versión revisada: 17-10-12, Aceptado: 21-11-12

Licencia Creative Commons BY NC ND · $2012 \cdot$ Asociación Española de Contabilidad y Administración de Empresas - AECA 
Los títulos de grado que comenzaron su andadura en el curso 2008/09, han culminado en el curso académico $2011 / 12$, con la presentación y defensa de un trabajo fin de grado. Aunque existen titulaciones con gran tradición en la elaboración de un trabajo o proyecto fin de carrera, otras titulaciones en las que tienen un peso importante las materias contables, tales como economía, administración y dirección de empresas, turismo y otras vinculadas a las ciencias sociales, no cuentan con tal tradición. Para estas titulaciones, el trabajo fin de grado supone un nuevo reto sobre el que muchas universidades en España están trabajando intensamente. El presente trabajo se enmarca en la normativa desarrollada al respecto por la Universidad Rey Juan Carlos y las experiencias de la Facultad de Ciencias del Turismo sobre el planteamiento del trabajo fin de grado en cuanto a las competencias, indicadores, agentes evaluadores e hitos de evaluación.

\section{EL TRABAJO DE FIN DE GRADO EN LA NORMATIVA DE LA UNIVERSIDAD REY JUAN CARLOS}

Según lo establecido en el Real Decreto 1393/2007, cada Universidad, en el ejercicio de su autonomía, podrá establecer las normas relativas a la elaboración, presentación y evaluación del Trabajo Fin de Grado (TFG).

En la Universidad Rey Juan Carlos(URJC) el trabajo fin de grado deberá ser realizado de forma individual por cada estudiante y deberá estar orientado a la aplicación de las competencias generales asociadas a la titulación, a capacitar para la búsqueda, gestión, organización e interpretación de datos relevantes, para emitir juicios que incluyan una reflexión sobre temas relevantes de índole social, científica, tecnológica o ética, y que facilite el desarrollo de un pensamiento y juicio crítico, lógico y creativo.

El contenido de cada TFG corresponde a uno de los siguientes tipos:

1. Trabajos experimentales relacionados con la titulación, que podrán desarrollarse en departamentos universitarios, laboratorios, y centros de investigación de la URJC, así como centros y empresas especializadas.

2. Trabajos de revisión e investigación bibliográfica centrados en diferentes campos relacionados con la titulación.

3. Otros trabajos que corresponderán a ofertas de profesores, no ajustadas a las modalidades anteriores, según se especifique en la normativa de cada Centro.

El TFG se lleva a cabo bajo la supervisión de un tutor académico que actúa como Director, y que es un docente a tiempo completo. Su labor consiste en orientar, asesorar y planificar las actividades del alumno, hacer un seguimiento del trabajo durante el periodo de duración del mismo y colaborar en todo aquello que permita una buena consecución del mismo. Para que pueda ser defendido, el tutor debe emitir una autorización.

Cada Centro nombra una Comisión de Trabajo Fin de Grado quien se encarga de gestionar las propuestas de trabajos de fin de grado por los profesores, asignar los temas a los alumnos, proponer las Comisiones de Evaluación del TFG y demás cuestiones relacionadas con la elección y asignación de trabajos.

La evaluación de los TFG se realiza por una Comisión de Evaluación, compuesta por dos profesores que imparten docencia en el centro y un miembro externo a la URJC, que podrá ser profesor de otra Universidad y pertenecer a alguno de los ámbitos de conocimiento de la titulación, o un experto de reconocido prestigio en el ámbito del que se trate. 
Según la normativa de la URJC, es la Comisión de Evaluación quien deliberará sobre la calificación de los TFG, teniendo en cuenta la documentación presentada y la exposición pública de los trabajos. Establece también esta normativa que la calificación global tendrá en cuenta la calidad científica y técnica del TFG, la calidad del material, la claridad expositiva, la capacidad de debate y la defensa argumental.

\section{EL TRABAJO DE FIN DE GRADO EN LOS ESTUDIOS DE GRADO EN TURISMO}

Los estudios de Grado en Turismo comenzaron a impartirse en la URJC en el curso 2008/09, de modo que en el curso 2011/12 se ha graduado la primera promoción de estudiantes. Según la memoria del Grado, el objetivo del TFG es verificar la adquisición por parte del estudiante de las destrezas y competencias descritas en los objetivos generales del título, junto a destrezas específicas de orientación profesional.

Así, al igual que en la normativa de la URJC, se incide en la aplicación de competencias genéricas. Establecido el marco general en que se articula el TFG en la URJC, serán los centros los que desarrollarán la normativa para adecuarla a las características propias de cada uno de los títulos de grado que se impartan en su centro (art. 2).

El objeto de este trabajo es, en primer lugar, seleccionar las competencias más importantes objeto de evaluación en el TFG y sus indicadores y, en segundo lugar, plantear la discusión sobre los agentes evaluadores, así como sobre los diferentes momentos o hitos de evaluación, considerando las circunstancias actuales de la universidad. Además, se incluyen los resultados sobre los primeros TFG defendidos, así como algunas reflexiones sobre el proceso global.

A pesar de que la URJC establece que el único agente evaluador es la Comisión Evaluadora, desde la Facultad de Ciencias del Turismo (y ante la posibilidad de que cada Centro gestione el TFG según sus características) se propuso la posibilidad de que en el proceso de evaluación participen indirectamente, además de la Comisión Evaluadora, los tutores de los TFG. Por ello, fue necesario debatir acerca de quiénes deberían ser los agentes evaluadores y cuál sería su papel en las diferentes fases del proceso de evaluación; junto con el conjunto de indicadores empleados para medir el grado de cumplimiento con el que el alumno alcanzaría las competencias objeto de evaluación por el trabajo fin de grado.

\section{PROPUESTA DE COMPETENCIAS, AGENTES EVALUADORES E HITOS O MOMENTOS DE EVALUACIÓN DEL TFG.}

El punto de partida para la definición de competencias objeto de evaluación del TFG fue la selección que ha realizado cada Facultad en la elaboración del plan de estudios. En la memoria del Grado en Turismo que se imparte en la URJC, se contemplaron 23 competencias transversales, extraídas del Título de Grado en Turismo publicado por la ANECA y en cuya elaboración participaron 47 universidades españolas, entre ellas la propia URJC.

Sin embargo, el hecho de que el trabajo fin de grado persiga integrar los contenidos formativos recibidos, las competencias específicas y muchas competencias transversales, no significa que sea necesario evaluar de nuevo todas las competencias específicas, así como el conjunto de competencias transversales definidas en el título. Por ello, se recomienda una selección priorizada con la finalidad de realizar una evaluación de competencias realista y operativa.

Para la definición de las competencias transversales asociadas al TFG, se diseñó un cuestionario que ha sido enviado a todos los profesores de la Facultad de Ciencias del 
Turismo. En este cuestionario se incluyó un listado con 23 ítems que se correspondían con las competencias transversales incluidas en la memoria del Grado en Turismo. Se solicitaba a los encuestados, que valorasen mediante una escala likert de 1 a 5 el grado de relevancia de cada una de las competencias transversales en le evaluación del TFG. Se incluyó, un ítem adicional para que los profesores opinaran acerca de quiénes deberían ser los agentes evaluadores del TFG.

El cuestionario se administró electrónicamente a los 130 profesores de la Facultad de Ciencias del Turismo. La encuesta fue respondida por 52 profesores, lo que muestra un grado de respuesta del $40 \%$. Sin embargo, es importante tener en cuenta que, que durante el curso académico 2011/12 no todos los profesores fueron tutores de TFG. De acuerdo con la normativa de la URJC, solo tienen obligatoriedad de tutorizar TFG los profesores con dedicación a tiempo completo.

Una vez recogidos y analizados los resultados se obtuvo información sobre dos aspectos clave:

1. Sobre las competencias transversales más relevantes para el TFG, los resultados reflejan que la comunicación oral y escrita en lengua nativa, la capacidad de gestión de la información; capacidad de análisis y síntesis, la capacidad de organización y planificación y el razonamiento crítico son las consideradas más importantes, habiendo obtenido una valoración media superior a 4,5 /(Tabla 1).

TAbla 1. Competencias transversales del GRAdo en tURismo valoradas por encima de 4,5 (SObre 5)

\begin{tabular}{lc}
\hline Comunicación oral y escrita en lengua nativa & $\mathbf{4 , 6 6}$ \\
\hline Capacidad de gestión de la información & 4,6 \\
\hline Capacidad de análisis y síntesis & 4,58 \\
\hline Capacidad de organización y planificación & 4,54 \\
\hline Razonamiento crítico & 4,54 \\
\hline
\end{tabular}

Fuente: elaboración propia

Como puede observarse, la mayoría de las competencias seleccionadas corresponden a competencias de carácter instrumental recogidas en el Proyecto Tuning (González et al., 2009), salvo la competencia razonamiento crítico, de carácter interpersonal y que en el proyecto anteriormente citado no aparece como tal, sino que es denominada capacidad crítica y autocrítica. Estas competencias consideradas como más relevantes en la evaluación del TFG, coinciden con las que obtuvieron Rullán et al. (2010) tras analizar las competencias más comúnmente consideradas evaluables en la materia TFG en los grados de Ciencias Sociales de la Universidad Autónoma de Barcelona. Estos autores observaron además que la priorización de las competencias no era la misma en todas las ramas que, además, pueden interpretar de forma distinta una misma competencia, otorgándole incluso distintos niveles de alcance.

Valderrama et al. (2009) también realizaron una encuesta para definir las competencias que deberían evaluarse en los TFG. Sin embargo, los estudios analizados eran ingenierías y los encuestados responsables académicos del ámbito de las ingenierías. Esto conlleva que los resultados no sean totalmente comparables. Aun así, entre las competencias transversales más votadas se encontraban la comunicación 
oral y escrita en lengua nativa, la capacidad de organización y planificación y la capacidad de análisis y de síntesis.

Mateo (2009) también realizó un estudio de las competencias más relevantes en la evaluación del TFG para el ámbito de las Ciencias Sociales. Sin embargo, en este caso la relevancia de las competencias se analizaba en cada una de las fases de elaboración del TFG propuesta en este trabajo, comenzando por la fase cero o momento 1 de "Elección del tema" y terminando en el momento 5 destinado a la "Evaluación, recomendaciones y orientaciones". En este caso las competencias tenían, por tanto, un mayor nivel de desglose otorgándoles una orientación u otra en función del momento o de la fase en que se encontraban. Por eso, en algunos casos más que competencias transversales podría hablarse de los propios indicadores aplicables a las mismas.

2. El segundo aspecto relevante es el relacionado con los agentes evaluadores del TFG. Con bastante frecuencia el docente está acostumbrado a ser el único evaluador de los alumnos. Sin embargo, la participación de diferentes agentes en el proceso de evaluación hace que éste se enriquezca al participar de forma conjunta en la medición del grado de desarrollo alcanzado por el alumno en relación a las competencias objeto de evaluación.

La opinión de los profesores encuestados en torno a quiénes deben ser los agentes evaluadores del TFG, es bastante significativa. Los profesores participantes han contestado mayoritariamente (un $68 \%$ de los casos) que tanto el tutor como la Comisión Evaluadora han de participar en el proceso de evaluación. El grado de opinión respecto a los profesores que consideran que sólo el tutor o sólo el tribunal deben ser los agentes evaluadores se sitúa en torno al 15\% y el 17\% respectivamente (llustración 1)

\section{ILUSTRACIÓN 1: Agentes EVALUADORES}

$$
\text { - Tutor y Tribunal } \quad \text { Tribunal } \quad \text { Tutor }
$$

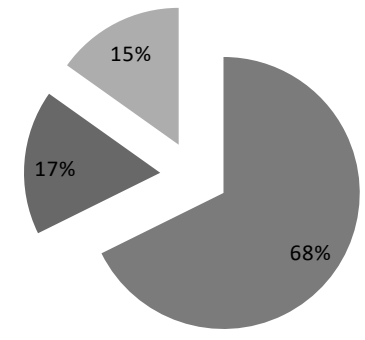

Fuente: elaboración propia

En el cuestionario no se incluyeron items relacionados con qué competencias debe evaluar cada uno de los agentes porque incrementaba notablemente la complejidad del mismo y el tiempo necesario para cumplimentarlo. No obstante, existe literatura al respecto, en la que basarse para estipular tanto los hitos y las competencias en las que deben participar los agentes evaluadores.

De la normativa de la URJC se desprende la participación de distintos agentes evaluadores, unos de forma explícita y otros de forma tácita. Por una parte, se encuentra el tutor o director del trabajo fin de grado. Aunque la normativa, no parece atribuirle directamente el calificativo de evaluador, ni de calificador, es requisito indispensable para la presentación del TFG que el tutor dé su autorización. 
El segundo agente evaluador es el tribunal o comisión evaluadora compuesta por tres miembros, siendo uno de ellos un profesional procedente de la industria 0 perteneciente a otra Universidad.

Quizás sea éste uno de los aspectos más discutibles del procedimiento que se ha asignado en esta Universidad en lo relativo al proceso de evaluación y calificación. Sin hacer un análisis exhaustivo, se han revisado los sistemas de evaluación de los TFG de otras universidades y se observa la gran diversidad de planteamientos. Además, es importante tener en cuenta que muchos centros han comenzado a implantar las titulaciones de grado recientemente y están en proceso de desarrollar el sistema de evaluación y calificación del TFG.

Del análisis efectuado, se observa que en unos casos es el tribunal el encargado de evaluar y calificar el TFG, siendo el tutor parte de este tribunal. Este caso es el que se aplica en la Facultad de Químicas de la Universidad de Burgos. En otras ocasiones es el tribunal el que evalúa y califica el TFG, sin que el tutor tome partido más allá de dar autorización para la presentación del trabajo. Este procedimiento es el que se aplica en la Universidad de Zaragoza, en la Facultad de CC. Físicas de la Universidad de Santiago y en la Universidad de Sevilla (Geografía, Filología y Química).

Existen otras posibilidades donde el tutor tiene un papel más activo. Así, en determinados centros es el tribunal el que califica el TFG, pero teniendo en cuenta el informe proporcionado por el tutor o director. Este es el sistema propuesto en algunos centros de la Universidad de Castilla la Mancha y de la Universidad de Salamanca.

Finalmente, y siguiendo el planteamiento anterior, algunas universidades han detallado el peso relativo que sobre la calificación final tendrá la evaluación del tutor y la del tribunal. En la Universidad de Valencia el 30\% de la evaluación corresponderá al tutor y el $70 \%$ a la comisión evaluadora o tribunal ante el cual el alumno presentará el TFG. En la Escuela de Estadística de la UCM, es el tribunal el que emite la calificación, pero basándose en un $20 \%$ en el informe emitido por el tutor.

El procedimiento adoptado en la URJC, prevé que el tutor o director del TFG emita una autorización para la lectura, donde el tutor dispone de un espacio para aportar observaciones y comentarios. Un posterior desarrollo de este apartado de "Observaciones y comentarios" podría dar lugar a que el tutor informara de su evaluación respecto a determinadas competencias transversales.

Además de los dos aspectos tratados previamente (competencias y agentes evaluadores) otro aspecto relevante en el proceso de evaluación consiste en definir en qué momento se evalúan los TFG y cuáles serán las evidencias que se pedirán al estudiante sobre las que hacer la evaluación. Esto es lo que se denomina como hitos del proceso de evaluación.

La definición de los distintos momentos o hitos en la evaluación del TFG planteada en algunos trabajos está justificada en la aplicación del proceso de evaluación continua seguido en el resto de asignaturas. Así, por ejemplo, Valderrama (2009) y Rullán et al (2010) distinguen tres momentos: inicio, seguimiento y final y desarrollan indicadores asociados a cada competencia y a cada uno de los hitos del proceso. Por otra parte, Mateo et al. (2009) distinguen cinco fases, puesto que incorporan una primera de elección y justificación del tema por parte de los alumnos y otra final donde se pide al alumno que efectúe un análisis crítico del proceso de aprendizaje seguido durante la elaboración del TFG.

Indudablemente, el planteamiento de distintos momentos de evaluación responde a los requerimientos de la evaluación continua y facilita a cada agente evaluador en cada momento los indicadores que debe utilizar para realizar la evaluación del TFG. A pesar de que esta metodología presenta todos los criterios deseables en cuanto a 
objetividad, transparencia y detalle, sería cuestionable sU aplicabilidad en la URJC por motivos relacionados con número de alumnos y profesores involucrados en el proceso'.

Tomando como referencia el reglamento de la URJC y las propuestas relacionadas con el TFG planteadas por otras universidades, desde la Facultad de Ciencias del Turismo se propuso un único hito de evaluación del trabajo fin de grado, donde tanto tutor como tribunal participen. De este modo, el tutor puede realizar la evaluación de todas las competencias en el momento en que se considera que el trabajo está finalizado, y el Tribunal puede realizar la evaluación en el momento en el que se le entrega el trabajo y se realiza la exposición oral. Por tanto, los agentes involucrados en el proceso son el Tribunal y el Tutor, ambos responsables de la evaluación de dos evidencias presentadas por el estudiante; el documento escrito del TFG y la presentación oral del mismo.

Sobre estas dos evidencias y en un solo momento de evaluación, puesto que el trabajo está concluido (fase final de entrega y exposición del TFG), el Tribunal emite una calificación sobre el trabajo considerando la evaluación previa del tutor. La evaluación previa del tutor consistiría en el consentimiento del mismo para la presentación del trabajo y la evaluación de competencias basada en los indicadores. A pesar de que la nota final del TFG es la proporcionada por el Tribunal, éste se basaría a su vez en la nota "orientativa" que previamente ha presentado el tutor del trabajo.

De este modo, tanto tutor como tribunal podían hacer uso de una misma plantilla de evaluación (ver Anexo I) con los indicadores asociados a cada competencia que les permiten realizar la evaluación correspondiente. Siempre se podría dejar la opción de no evaluar por motivos relacionados a que puede que "no aplique" medir un indicador concreto, pero eso dependerá de las circunstancias concretas de cada trabajo.

Desde la FCT se propuso como herramienta de evaluación un documento o ficha donde, tanto tutor como Comisión Evaluadora, pueden establecer la nota obtenida por el estudiante para cada una de las cinco competencias detectadas como más relevantes, basándose en los indicadores asociados a las mismas, que permiten una evaluación en una escala de 1 a 5. Los indicadores han sido clasificados por competencias con base a lo realizado previamente diversos autores (Valderrama et al, 2009 y Rullán et al. 2010)

Aun así, para culminar el proceso, sería conveniente desarrollar el grado de cumplimiento de cada indicador que se corresponde a cada calificación de 1 a 5 (por medio del desglose de rúbricas).

\section{EXPERIENCIAS ACUMULADAS/OBTENIDAS DEL PROCESO DE ASIGNACIÓN Y EVALUACIÓN DE LOS TFG DURANTE EL CURSO 2011/12.}

Finalizado el curso académico 2011/12 en el que se han defendido 91 TFG en la Facultad de Ciencias del Turismo, se dispone de información suficiente para realizar una descripción y evaluación de las experiencias adquiridas en este proceso en torno a:

- La propuesta y asignación de temas para el desarrollo del TFG.

- El trabajo desarrollado por el tutor del TFG.

- La evaluación de los TFG.

\footnotetext{
1 El curso 2011/2012 se estimó que habrá 440 alumnos matriculados en la materia TFG
} 


\subsection{LA PROPUESTA Y ASIGNACIÓN DE TEMAS PARA El DESARROLlO DEL TFG.}

El proceso relativo a la propuesta y asignación de temas para el curso 2011/12 comenzó en el curso académico anterior (2010/11) con objeto de que los temas estuvieran asignados con la suficiente antelación para que tanto alumnos como tutores pudieran desarrollar su trabajo y defender el tema en el curso 2011/12. Tras considerar el número de alumnos que estarían matriculados en $4^{\circ}$ curso en el año siguiente y el número de profesores a tiempo completo con docencia en esta Facultad, en abril de 2011 se solicitó a este colectivo de profesores que propusieran al menos tres temas para los TFG. Aquellos profesores con docencia en el Grado en Turismo en inglés debían proponer temas que fueran desarrollados y defendidos en este idioma. También existía la posibilidad de que un tema pudiera ser elegido por hasta dos alumnos aunque desarrollado individualmente, claro está, como establece el reglamento del TFG. Además, una tercera opción, consistió en que alumnos y profesores tras llegar a un acuerdo previo, comunicaban a la Comisión de TFG sU deseo de trabajar en un tema acordado entre ellos.

Una vez recibidos y revisados los temas propuestos por los profesores, la Comisión de TFG hizo pública dicha relación de temas por medio de la página web de la Facultad para que, del 1 al 15 de junio, los alumnos realizasen sus elecciones indicando, en un documento diseñado a tal efecto y que debían remitir a la Comisión, los diez temas seleccionados por orden de preferencia, el número de créditos superados y la nota media del expediente hasta ese momento.

Ordenados los alumnos por nota media de expediente, se realizaron las asignaciones de los temas. Se publicó en la página web de la Facultad el listado provisional de asignaciones $y$, tras un periodo de reclamaciones, a comienzos del mes de julio se publicó el listado definitivo indicando los temas y el tutor asignado y el alumno vinculado a ese tema y tutor. En este listado también se incluyeron aquellos alumnos que no habían seguido este proceso por haber llegado a un acuerdo previo con un profesor para que dirigiera un tema acordado entre ellos.

Este proceso se realizó de nuevo en el mes de enero, según está previsto en el Reglamento de TFG. Se solicitaron temas a aquellos profesores que, por distintas circunstancias, no los habían propuesto en el mes de mayo y se publicó un nuevo listado de temas incluyendo los que habían quedado vacantes en junio así como las nuevas propuestas. Aquellos alumnos que no solicitaron tema en junio o que solicitaron temas que resultaron estar ocupados por otros compañeros con mejor nota de expediente, acudieron a esta nueva convocatoria. Se tomaron las mismas acciones que han sido descritas anteriormente y en el mes de febrero, se publicaron las nuevas asignaciones. Entre estas dos convocatorias se asignaron un total de 336 trabajos.

En abril de 2012, se iniciaba de nuevo el proceso de cara al curso 2012/13. Así, los profesores debían ofertar una nueva relación de temas para los alumnos que ya estaban en disposición de elegir. Sin embargo, la complejidad de este proceso se ha incrementado de forma importante. Esto ha sido debido a que la mayoría de las titulaciones de grado que se imparten en la URJC se implantaron en el curso 2009/10 y, por tanto, en el curso 2012/13 llega a cuarto curso la primera promoción de alumnos de estas titulaciones. Esto ha motivado, no sólo la incorporación de un importante número de alumnos a este proceso; sino también, que profesores deban aportar temas para que sean elegidos por los alumnos de las distintas titulaciones en la que imparten docencia.

Por ello, en abril de 2012, el proceso de solicitud de temas a profesores y de oferta y asignación a los alumnos se ha realizado de forma centralizada por parte de la recientemente creada Unidad de Trabajo de Fin de Grado (dependiente del 
Vicerrectorado de Ordenación Académica). Así, desde esta unidad se solicitó a los profesores que propusieran temas que pudieran ser elegidos por 6 estudiantes, con la posibilidad de ofertar entre 2 y 6 temas y que cada tema pudiera ser elegido entre 3 y un estudiante. Del mismo modo que en el curso anterior, finalmente los temas serán asignados teniendo en cuenta el orden de preferencia establecido por el alumno y la nota media de expediente de éstos. Esta asignación quedará formalizada a través de la firma por parte del profesor-tutor y del alumno de un documento donde se indica el título del tema del TFG que vincula a ambos.

\subsection{El TRABAJO DESARROLLADO POR EL TUTOR}

Del total de los 336 trabajos asignados por la Facultad de Ciencias del Turismo (FCT) entre junio de 2011 y enero del 2012, fueron defendidos 91 TFG, lo que representa que un $27 \%$ de los trabajos inicialmente asignados.

Con el objeto de recoger información relativa a la dedicación de los tutores de TFG; a las razones que han dificultado la defensa de un mayor número de trabajos; a la evaluación realizada por los mismos en torno al grado de consecución de las competencias transversales consideradas más relevantes (y a las que hemos hecho referencia anteriormente); así como a los aspectos y acciones que estos mismos tutores consideran podrían mejorar los resultados obtenidos por los estudiantes; se diseño y administró un cuestionario electrónico.

Este cuestionario se ha distribuido por correo electrónico a todos aquellos tutores de TFG cuyos alumnos ya habían defendido algún trabajo. La tasa de respuesta ha sido de más de la mitad, concretamente del $58 \%$ y los resultados del mismo se exponen en los párrafos siguientes.

Las razones que han aportado los tutores para justificar la no defensa del total de los trabajos inicialmente asignados están básicamente relacionadas con la falta de tiempo dedicado por el estudiante (por la realización de las prácticas externas $u$ otros motivos), o porque los estudiantes estaban cursando alguna beca (principalmente Erasmus) en el extranjero y no les fue posible culminar con éxito el trabajo.

Un aspecto especialmente interesante es el relacionado con el tiempo dedicado por cada tutor a cada TFG asignado. Según el reglamento de TFG de la URJC, la labor del tutor del TFG consiste en orientar, asesorar y planificar las actividades del alumno, hacer un seguimiento del trabajo realizado durante el periodo de duración del trabajo a desarrollar y colaborar en todo aquello que permita una buena consecución del mismo según se detalla en la normativa desarrollada por la Universidad.

Según la información recogida del cuestionario, un $44 \%$ de los profesores encuestados le han dedicado a cada TFG entre 10 y 15 horas en concepto de tutorías y el $29 \%$ más de 16 horas. En cuanto al tiempo dedicado en revisión y corrección de trabajos hasta el visto bueno, el 33\% de los tutores le dedicaron a cada trabajo defendido más de 16 horas y el $39 \%$ entre 10 y 15 horas.

Considerando el tiempo dedicado a tutorías con el alumno, lectura y corrección de los distintos borradores hasta la versión definitiva puede afirmarse, según las respuestas de los tutores de TFG, que en casi el $30 \%$ de los caso asciende a más de 32 horas por trabajo; y en más del $40 \%$ de los casos a entre 20 y 30 horas de dedicación por TFG defendido. Si esto se multiplica por el número de TFG asignados a los profesores (media de 4 durante el curso 2011/2012), podemos fácilmente hacernos una idea de la importante carga de trabajo para el profesor y el número de créditos que se podría equiparar a la misma. Sin embargo, la carga docente reconocida a los profesores por TFG asignado es de cero, bien haya sido defendido por el alumno o se encuentre pendiente (llustración 2). 
ILUSTRACIÓN 2: DEDICACIÓN EN HORAS DE LOS TUTORES
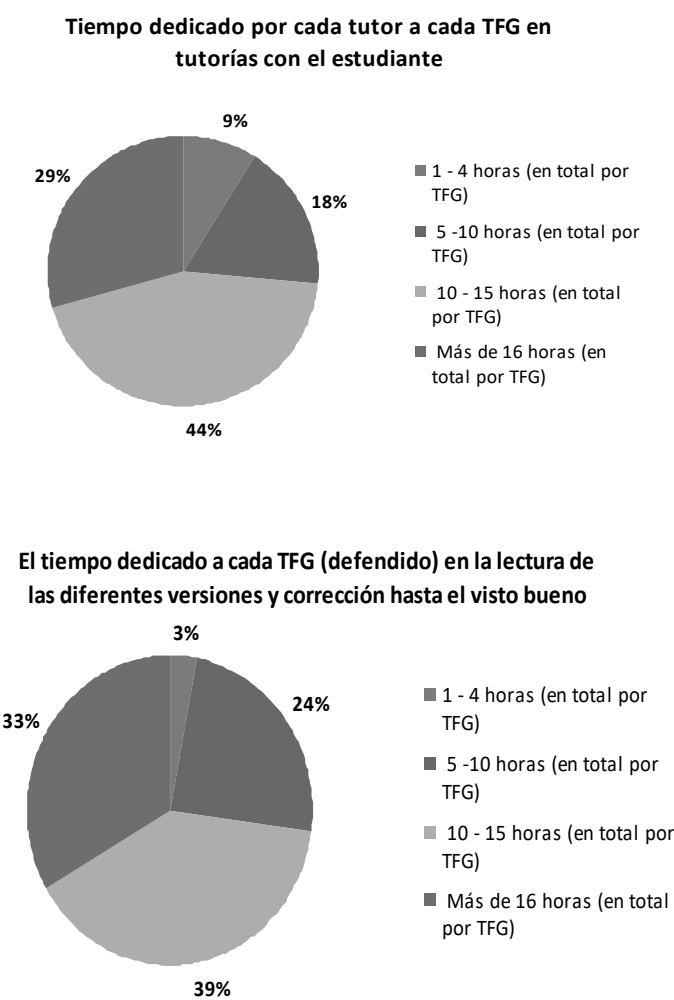

Fuente: Elaboración propia

Además, los alumnos que tienen un tema y tutor asignados y no defienden su TFG en el curso correspondiente, continúan con ese mismo tema y tutor (salvo causas excepcionales) hasta que la defensa tenga lugar, lo que puede generar para los tutores una acumulación de TFG de un curso para el siguiente, que le genere una carga de trabajo extraordinaria.

\subsection{EvaluACIÓN DE LOS TFG.}

Como se ha mencionado con anterioridad, desde la Facultad de Ciencias del Turismo se propuso un único hito de evaluación del trabajo fin de grado, donde tanto tutor como tribunal participasen. Sin embargo, la participación del tutor a través de la cumplimentación del Anexo I, ha sido muy reducida y, por ello, los resultados no se exponen en este documento. Esto ha sido debido principalmente a que la evaluación del tutor era de carácter voluntario. Según ha quedado anteriormente expuesto, la normativa de la URJC establece que la evaluación corresponde exclusivamente al tribunal. La nota media de los 91 trabajos presentados y defendidos en el curso $2011 / 2012$ fue de 8,1 sobre 10 puntos.

La calificación mayoritaria fue la de notable (considerando notable las calificaciones entre 7 y 8,9 puntos sobre 10), obtenida por el $53 \%$ de los TFGs defendidos. El $29 \%$ de los trabajos obtuvieron la calificación de sobresaliente (evaluados entre 9 y 9,9 puntos sobre 10) y el $7 \%$ de los trabajos presentados obtuvieron la calificación de Matrícula de Honor (10 sobre 10) como podemos ver llustración 3. 
ILUSTRACIÓN 3: CALIFICACIONES OBTENIDAS POR LOS ALUMNOS

\section{Notas obtenidas por los TFGs defendidos}

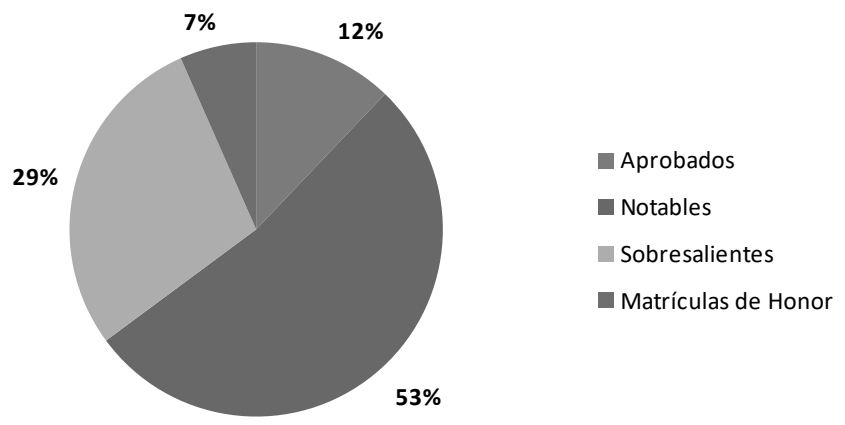

Fuente: Elaboración propia

En el cuestionario administrado se incluyeron 5 ítems para conocer la opinión de los tutores sobre el grado de consecución por parte de los estudiantes de las 5 competencias transversales detectadas como más relevantes. Si bien la nota media de los TFG defendidos puede ser considerada más que aceptable, la opinión de los tutores sobre el grado de "alcance" de las cinco principales competencias transversales revela que las capacidades asociadas a estas competencias no se han alcanzado de forma tan satisfactoria.

Tal y como puede observarse en la siguiente figura, la capacidad de comunicación escrita en lengua nativa por parte de los estudiantes es la capacidad en la que mejor se han desempeñado (el desempeño se debía valorar en una escala de 1 a 5, donde 1 implica que la competencia no ha sido alcanzada y 5 totalmente alcanzada), mientras que la capacidad de razonamiento crítico es la que menor grado de consecución ha obtenido por los estudiantes que defendieron satisfactoriamente su TFG (valorada por los tutores con un 3,3 sobre 5) (llustración 4).

\section{ILUSTRACIÓN 4: GRADO DE ALCANCE DE LAS PRINCIPALES COMPETENCIAS TRANSVERSALES POR LOS ESTUDIANTES QUE DEFENDIERON SU TFG EN 2011/12 (ESCALA 1 A 5)}

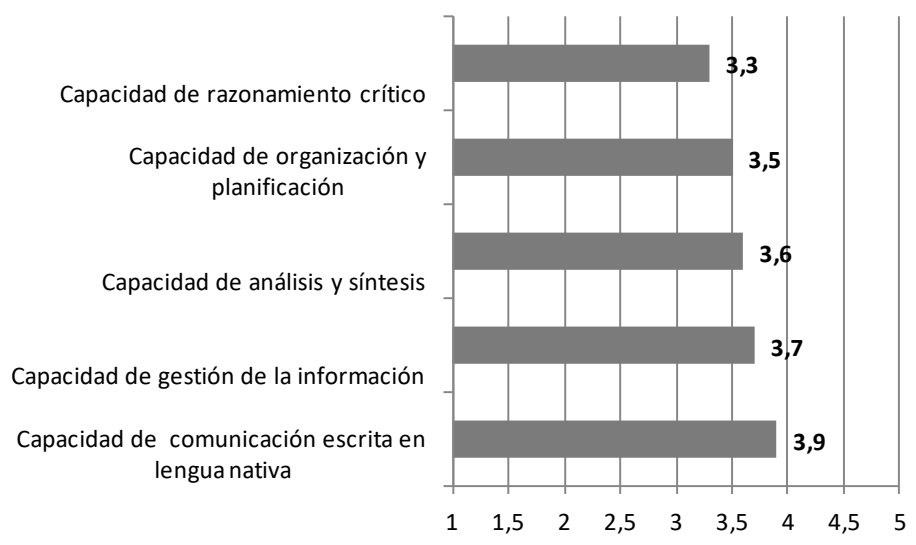

Fuente: Elaboración propia 
Finalmente, el cuestionario sondeaba la opinión de los tutores su opinión respecto a las medidas se deberían adoptar para que los estudiantes alcanzaran de modo óptimo las competencias anteriormente mencionadas. Como puede observarse en el siguiente gráfico, el $65 \%$ de los tutores encuestados apuestan porque la universidad desarrolle módulos o cursos específicos destinados a los estudiantes, para que estos alcancen de un modo más satisfactorio las competencias transversales (llustración 5).

\section{ILUSTRACIÓN 5: OPINIÓN DE LOS PROFESORES RESPECTO A DISTINTOS MEDIOS PARA DESARROLLAR COMPETENCIAS TRANSVERSALES}

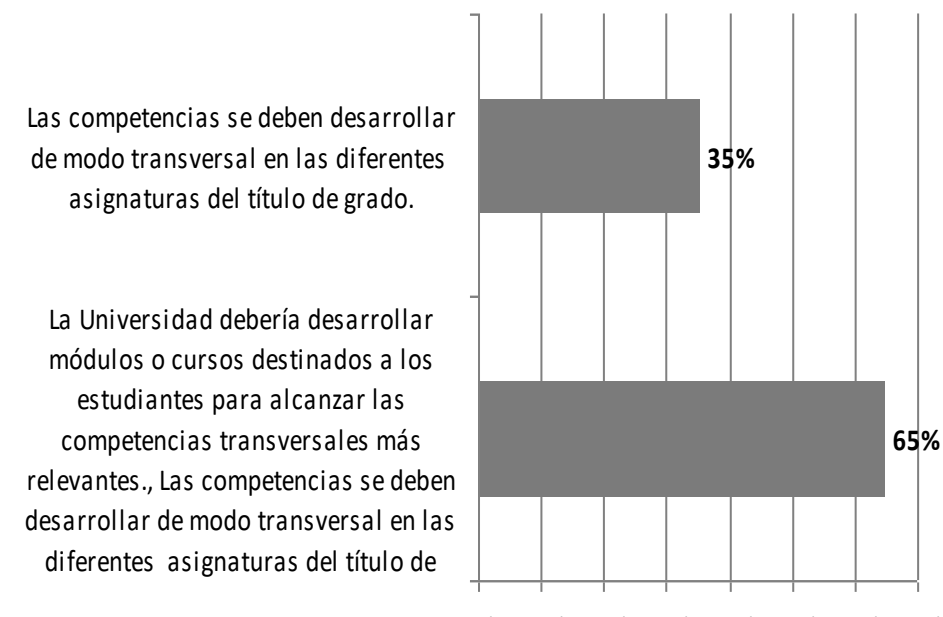

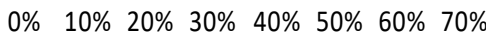

Fuente: Elaboración propia

\section{DISCUSIONES Y CONCLUSIONES}

El Trabajo Fin de Grado está presentando, en muchas Facultades y Centros, un nuevo reto en cuanto al modo de gestión y, sobre todo, en lo relacionado con la metodología de evaluación del mismo. No existe consenso en cuanto a la forma de evaluación del TFG, existiendo diferentes propuestas de evaluación de competencias, indicadores e hitos.

En la Universidad Rey Juan Carlos el TFG está orientado a la aplicación de las competencias generales de cada titulación. Para determinar qué competencias (de las 23 detalladas en la Memoria del Titulo de Grado en Turismo) son las más relevantes para el TFG del Título de Grado en Turismo, se diseñó un cuestionario que fue administrado electrónicamente entre el profesorado de la Facultad durante el curso 2011/2012. Las cinco competencias más relevantes para los profesores encuestados fueron comunicación y escrita en lengua nativa, la capacidad de gestión de la información; capacidad de análisis y síntesis, la capacidad de organización y planificación y el razonamiento crítico.

Los indicadores asociados a cada competencia han sido extraídos de estudios ya existentes y facilitan la evaluación del TFG.

En cuanto a los agentes evaluadores del TFG, existen diversas posturas. Hay universidades que apuestan por un solo agente evaluador (Tribunal), mientras que hay otras en las que son el Tutor del trabajo y el Tribunal los agentes evaluadores del TFG. Partiendo del reglamento establecido por la URJC, se propone que sea el Tribunal al que se presenta el TFG el principal agente evaluador, a pesar de que más de la mitad de los profesores encuestados para este trabajo consideran que deben ser Tutor y 
Tribunal los dos agentes evaluadores. Para considerar además la evaluación del Tutor, se ha diseñado una plantilla que sirva como herramienta de evaluación tanto al tutor como al Tribunal, de modo que, aunque solamente la nota del Tribunal será la definitiva, el Tribunal puede apoyarse y orientarse en las notas aportadas por el tutor para cada competencia e indicador. De esta forma, aunque solamente figura la nota de un agente evaluador, han participado indirectamente en el proceso los dos agentes principales detectados por los encuestados.

Sobre los hitos o momentos de evaluación existen también diferentes propuestas. A pesar de que la diferenciación de "fases de evaluación" es más coherente con el proceso de evaluación continua, en el presente trabajo se propone un momento de evaluación del documento escrito correspondiente al TFG y a la presentación oral del mismo. Esta propuesta es la que se enmarca en el reglamento sobre el TFG elaborado por la URJC.

La defensa de los primeros 91 trabajos en la Facultad de Ciencias del Turismo ofrece interesantes resultados que invitan al debate y que entenderemos evolucionarán en los próximos años.

Sólo se han defendido un $27 \%$ de los trabajos asignados. Es la primera promoción de alumnos que han llegado a cuarto curso del Grado en Turismo y, sin duda, esto ha podido influenciar a aquellos alumnos y tutores menos decididos y que han preferido recibir información adicional a través de las experiencias de sus compañeros. Sin embargo, si esta tendencia se mantiene, el periodo de finalización del grado superaría en la mayoría de los casos los cuatro cursos académicos.

Otro aspecto que indudablemente debe ser abordado es el reconocimiento de la carga de trabajo del profesorado. La organización de cursos o seminarios dirigidos a los alumnos a mejorar el grado de alcance de las competencias transversales puede aliviar en parte el trabajo desarrollado por los tutores. Pero, aún así, es difícilmente entendible que este trabajo no esté reconocido en la carga docente del profesor. Sin embargo, la situación económica actual y la continua reducción del presupuesto destinado a las Universidades en nuestra Comunidad, no son especialmente alentadoras en este sentido.

Objeto de un análisis más profundo y de la recogida de información más detallada debería ser la discrepancia existente entre las calificaciones obtenidas por los alumnos en sus trabajos y la valoración realizada por los profesores del grado de consecución de los alumnos de las competencias transversales.

Los tribunales han valorado de forma más positiva los trabajos evaluados que los propios tutores. Desde nuestra experiencia en este proceso, esto es un indicador del importante compromiso de la mayoría de los profesores en las labores de tutorización quienes han conseguido que sus alumnos finalicen trabajos de forma satisfactoria gracias a las continuas tutorías y revisiones de los documentos.

Sin embargo, este grado de dedicación a los alumnos es difícilmente sostenible. En el curso 2011/12 se asignaron hasta cuatro trabajos a cada tutor y en el 2012/13 se pretende asignar hasta seis tutorandos por profesor que se acumularán a las asignaciones anteriores que no hayan sido defendidas, lo que incrementará indudablemente el tiempo de dedicación del profesorado a labores no reconocidas dentro de su carga docente y que se suma a los incrementos en la docencia que las circunstancias actuales están provocando en la universidad pública. 


\section{BIBLIOGRAFÍA}

MATEO, J. (COORD.) (2009): Guía para la evaluación de competencias en el trabajo de fin de grado en el ámbito de las ciencias sociales y jurídicas. Barcelona: Agència per a la Qualitat del Sistema Universitari de Catalunya.

Rullán, M., Fernández-Rodríguez, M., Estapé, G., Márquez, M. D., (2010): La evaluación de competencias transversales en la materia trabajos de fin de grado. Un estudio preliminar sobre la necesidad y oportunidad de establecer medios e instrumentos por ramas de conocimiento. Revista de Docencia Universitaria, 8(1): 74-100.

UnIVERSIDAd Rey JuAn Carlos (2011). Reglamento del Trabajo Fin de Grado. Disponible en:http://www.urjc.es/alumnos/normativa/archivos/Normativa\%20Trab ajo\%20Fin\%20de\%20Grado.pdf

VALDERRAMA, E. (COORD.) (2009): Guía para la evaluación de competencias en los trabajos fin de grado y de máster en las ingenierías. Barcelona: Agència per a la Qualitat del Sistema Universitari de Catalunya.

GONZÁlEZ, J. y WAGENAAR, R. (COORDS.) (2009): Una introducción a Tuning Educational Structures in Europe. La contribución de las Universidades al proceso de Bolonia. Universidad de Deusto. Disponible en: http://www.unideusto.org/tuningeu/images/stories/documents/Genera Brochure Spanish version.pdf 


\section{ANEXO 1. PLANTILLA PARA LA EVALUACION DEL TFG}

C1. C1. Comunicación oral y escrita en lengua nativa: Capacidad para transmitir en público información, ideas, problemas y soluciones; y capacidad para redactar informes o documentos y hacerse entender.

\begin{tabular}{|c|c|c|c|c|c|c|c|c|c|c|c|c|c|}
\hline \multirow[b]{2}{*}{ ICl.1. } & \multirow{2}{*}{$\begin{array}{l}\text { INDICADORES } \\
\begin{array}{l}\text { Expone ideas y conceptos de forma } \\
\text { comprensible }\end{array}\end{array}$} & \multicolumn{6}{|c|}{ EVALUACIÓN TUTOR } & \multicolumn{6}{|c|}{$\begin{array}{l}\text { EVALUACIÓN } \\
\text { TRIBUNAL }\end{array}$} \\
\hline & & 1 & 2 & 3 & 4 & 5 & NA & 1 & 2 & 3 & 4 & 5 & NA \\
\hline IC 1.2. & $\begin{array}{l}\text { Emplea vocabulario adecuado en } \\
\text { cada circunstancia }\end{array}$ & 1 & 2 & 3 & 4 & 5 & NA & 1 & 2 & 3 & 4 & 5 & NA \\
\hline IC1.3. & $\begin{array}{l}\text { En exposición oral: muestra empatía } \\
\text { con la audiencia, tono de voz y } \\
\text { volumen adecuados. }\end{array}$ & 1 & 2 & 3 & 4 & 5 & NA & 1 & 2 & 3 & 4 & 5 & NA \\
\hline IC 1.4. & $\begin{array}{l}\text { Durante el discurso, liga bien los } \\
\text { conceptos, comunicando } \\
\text { coherentemente ideas propias y } \\
\text { ajenas. }\end{array}$ & 1 & 2 & 3 & 4 & 5 & NA & 1 & 2 & 3 & 4 & 5 & NA \\
\hline IC 1.5. & $\begin{array}{l}\text { Escribe correcta y claramente, } \\
\text { utilizando normas } \\
\text { adecuadas. }\end{array}$ & 1 & 2 & 3 & 4 & 5 & NA & 1 & 2 & 3 & 4 & 5 & NA \\
\hline
\end{tabular}

\begin{tabular}{|c|c|c|c|c|c|c|c|c|c|c|c|c|c|}
\hline \multirow{3}{*}{$\begin{array}{l}\text { C2. } \\
\text { IC2.1. }\end{array}$} & \multicolumn{13}{|c|}{$\begin{array}{l}\text { C2. Capacidad de gestión de la información: Tiene habilidad para buscar, reunir y } \\
\text { analizar información proveniente de fuentes diversas. }\end{array}$} \\
\hline & \multirow{2}{*}{$\begin{array}{l}\text { INDICADORES } \\
\text { Lleva a cabo una búsqueda de } \\
\text { información inicial sobre el tema } \\
\text { escogido }\end{array}$} & \multicolumn{6}{|c|}{ EVALUACIÓN TUTOR } & \multicolumn{6}{|c|}{$\begin{array}{l}\text { EVALUACIÓN } \\
\text { TRIBUNAL }\end{array}$} \\
\hline & & 1 & 2 & 3 & 4 & 5 & NA & 1 & 2 & 3 & 4 & 5 & NA \\
\hline IC2.2. & $\begin{array}{l}\text { Selecciona las fuentes fundamentales } \\
\text { para la construcción del marco } \\
\text { referencial o teórico del TFG }\end{array}$ & 1 & 2 & 3 & 4 & 5 & NA & 1 & 2 & 3 & 4 & 5 & NA \\
\hline IC2.3. & $\begin{array}{l}\text { Relaciona la información extraída de las } \\
\text { fuentes con el propio planteamiento del } \\
\text { TFG }\end{array}$ & 1 & 2 & 3 & 4 & 5 & NA & 1 & 2 & 3 & 4 & 5 & NA \\
\hline IC2.4. & Interpreta rigurosamente la información & 1 & 2 & 3 & 4 & 5 & NA & 1 & 2 & 3 & 4 & 5 & NA \\
\hline
\end{tabular}


C3. C3. Capacidad de análisis y síntesis: Capacidad para descomponer situaciones complejas en sus partes constituyentes para conocer sus principios o elementos y sus relaciones.

\begin{tabular}{|c|c|c|c|c|c|c|c|c|c|c|c|c|c|}
\hline & INDICADORES & & $A L$ & $A C$ & 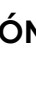 & TU1 & & & 20 & & & & \\
\hline IC3.1. & $\begin{array}{l}\text { Identifica las partes fundamentales del } \\
\text { proyecto, dibujando un diagrama a nivel } \\
\text { de bloques que las describe, visualizando } \\
\text { las relaciones entre ellas. }\end{array}$ & 1 & 2 & 3 & 4 & 5 & NA & 1 & 2 & 3 & 4 & 5 & NA \\
\hline IC3.2. & $\begin{array}{l}\text { Identifica los conocimientos implicados } \\
\text { en la base de la resolución del proyecto, } \\
\text { tanto sean propios de la disciplina como } \\
\text { ajenos }\end{array}$ & 1 & 2 & 3 & 4 & 5 & NA & 1 & 2 & 3 & 4 & 5 & NA \\
\hline IC3.3. & $\begin{array}{l}\text { Evalúa los resultados del proyecto } \\
\text { comparándolos con resultados similares } \\
\text { procedentes de fuentes ... }\end{array}$ & 1 & 2 & 3 & 4 & 5 & NA & 1 & 2 & 3 & 4 & 5 & NA \\
\hline IC3.4. & $\begin{array}{l}\text { Sintetiza la información obtenida y los } \\
\text { conocimientos propios en una visión } \\
\text { global y estructurada del "estado del } \\
\text { arte" del tema propuesto. }\end{array}$ & 1 & 2 & 3 & 4 & 5 & NA & 1 & 2 & 3 & 4 & 5 & NA \\
\hline
\end{tabular}

\begin{tabular}{|c|c|c|c|c|c|c|c|c|c|c|c|c|c|}
\hline \multirow{3}{*}{$\begin{array}{l}\text { C4. } \\
\\
\text { IC } 4.1 .\end{array}$} & \multicolumn{13}{|c|}{$\begin{array}{l}\text { C4. Capacidad de organización y planificación; pretende fijar los objetivos a lograr, } \\
\text { ordenar las prioridades en diferentes fases, determinar los medios más adecuados para } \\
\text { la consecución de los objetivos fijados y asegurar la efectiva aplicación de los mismos }\end{array}$} \\
\hline & \multirow{2}{*}{$\begin{array}{l}\text { INDICADORES } \\
\text { El estudiante es capaz de estructurar } \\
\text { las etapas del proyecto }\end{array}$} & \multicolumn{6}{|c|}{ EVALUACIÓN TUTOR } & \multicolumn{6}{|c|}{$\begin{array}{l}\text { EVALUACIÓN } \\
\text { TRIBUNAL }\end{array}$} \\
\hline & & 1 & 2 & 3 & 4 & 5 & NA & 1 & 2 & 3 & 4 & 5 & NA \\
\hline IC 4.2 & $\begin{array}{l}\text { El estudiante es capaz de definir la } \\
\text { metodología más oportuna para } \\
\text { desarrollar las etapas del trabajo. }\end{array}$ & & & & & & & & & & & & \\
\hline IC4.3. & $\begin{array}{l}\text { El estudiante es capaz de temporalizar } \\
\text { correctamente las fases del TFG }\end{array}$ & 1 & 2 & 3 & 4 & 5 & NA & 1 & 2 & 3 & 4 & 5 & NA \\
\hline IC4.4. & $\begin{array}{l}\text { El estudiante analiza el grado de } \\
\text { cumplimiento final de la planificación } \\
\text { inicial, las causas de eventuales } \\
\text { desviaciones y las consecuencias de } \\
\text { éstas. }\end{array}$ & 1 & 2 & 3 & 4 & 5 & NA & 1 & 2 & 3 & 4 & 5 & NA \\
\hline
\end{tabular}




\begin{tabular}{|c|c|c|c|c|c|c|c|c|c|c|c|c|c|}
\hline \multirow[t]{2}{*}{ C5. } & \multicolumn{13}{|c|}{$\begin{array}{l}\text { C5. Razonamiento crítico (en muchos sitios se llama razonamiento crítico y autocrítico, } \\
\text { de ahí los indicadores que aparecen y por esos habla de críticas de ideas propias) } \\
\text { Actitud reflexiva con el objeto de conocer la naturaleza de un problema identificando } \\
\text { incoherencias y carencias importantes (ya se trate de ideas propias o ajenas) }\end{array}$} \\
\hline & INDICADORES & & $A L$ & AC & ÓN & IU' & & & $\begin{array}{ll}A L U \\
B U N\end{array}$ & & & & \\
\hline IC5.1. & $\begin{array}{l}\text { El alumno se plantea preguntas del } \\
\text { tipo ¿qué pasaría się ¿qué hubiera } \\
\text { pasado si? }\end{array}$ & 1 & 2 & 3 & 4 & 5 & NA & 1 & 2 & 3 & 4 & 5 & NA \\
\hline IC5.2. & $\begin{array}{l}\text { Identifica los puntos fuertes y débiles } \\
\text { de un trabajo propio o ajeno. }\end{array}$ & 1 & 2 & 3 & 4 & 5 & NA & & 2 & 3 & 4 & 5 & NA \\
\hline IC5.3. & $\begin{array}{l}\text { Define soluciones propuestas mediante } \\
\text { argumentos lógicos y coherentes, } \\
\text { indicando si los argumentos están } \\
\text { contrastados con informaciones fiables } \\
\text { y referenciando fuentes. }\end{array}$ & 1 & 2 & 3 & 4 & 5 & NA & & 2 & 3 & 4 & 5 & NA \\
\hline IC5.4. & $\begin{array}{l}\text { Explica y argumenta si está en } \\
\text { condiciones de sacar adelante el } \\
\text { proyecto tal y como está planteado. }\end{array}$ & 1 & 2 & 3 & 4 & 5 & NA & & 2 & 3 & 4 & 5 & NA \\
\hline
\end{tabular}

